\title{
Pensamiento crítico en la escuela: una revisión de la literatura
}

\section{Critical thinking at school: a review of the literature}

\author{
María Juliana Ozaeta ${ }^{1}$ \\ María Paula Arias² \\ Laura Biviana Ortíz ${ }^{3}$ \\ Claudia Alejandra Duque-Romero" \\ Yenny Marcela Sánchez ${ }^{5}$ \\ Laura Yamile Henao-Morales ${ }^{6}$
}

\begin{abstract}
()
Recepción: 29/09/2021

Aprobación: 07/12/2021

Publicación: 22/12/2021

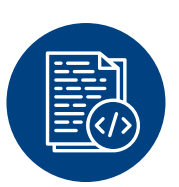

Para citar este artículo

Ozaeta, M. J., Arias, M. P., Ortíz, L. B., Duque-Romero, C. A., Sánchez, Y. M., \& Henao-Morales, L. Y. (2021). Pensamiento crítico en la escuela: una revisión de la literatura. Indagare, (9), 5-23. https://doi.org/10.35707/indagare/901
\end{abstract}

\footnotetext{
1 Semillero EDUCERE del Grupo de investigación Estudios en Educación (GESE), Universidad de Ibagué, Ibagué, Colombia. ORCID: 0000-0001-8285-4357. Correo electrónico: 3220182043@estudiantesunibague.edu.co

2 Semillero EDUCERE del Grupo de investigación Estudios en Educación (GESE), Universidad de Ibagué, Ibagué, Colombia. ORCID: 0000-0002-9093-1444. Correo electrónico: 3220162032@estudiantesunibague.edu.co

3 Semillero EDUCERE del Grupo de investigación Estudios en Educación (GESE), Universidad de Ibagué, Ibagué, Colombia. ORCID: 0000-0001-5521-2594. Correo electrónico: 3220162024@estudiantesunibague.edu.co

${ }^{4}$ Grupo de investigación Estudios en Educación (GESE), Universidad de Ibagué, Ibagué, Colombia. ORCID: 0000-0002-8596-2406. Correo electrónico: alejandra.duque@unibague.edu.co

5 Grupo de investigación Estudios en Educación (GESE), Universidad de Ibagué, Ibagué, Colombia. ORCID: 0000-0002-8634-2030. Correo electrónico: yenny.sanchez@unibague.edu.co

${ }^{6}$ Grupo de investigación Estudios en Educación (GESE), Universidad de Ibagué, Ibagué, Colombia. ORCID: 0000-0002-4801-0561. Correo electrónico: laura.henao@unibague.edu.co
} 


\title{
Resumen
}

Este artículo se deriva del proyecto titulado "Implementación de una estrategia de apropiación social de la CTeI (Ciencia, Tecnología e Innovación) que promueve el pensamiento crítico y creativo en niños, niñas y jóvenes de las instituciones educativas del Tolima”. Se planteó como propósito analizar la evidencia empírica y teórica disponible sobre el desarrollo del pensamiento crítico, a partir de estrategias metodológicas utilizadas en el aula con estudiantes de básica primaria y secundaria. Lo anterior, tras considerar que el pensamiento crítico aumenta la participación de la comunidad educativa para el reconocimiento y solución de problemas relacionados con el territorio que se habita. Por lo tanto, es tarea de la escuela promoverlo desde los primeros años; sin embargo, hay estudios que muestran avances en el desarrollo del pensamiento crítico y creativo, pero se concentran en la educación superior, mientras hay rezagos en primaria y secundaria. Finalmente, las investigaciones revisadas posicionan el papel de la escuela como escenario clave para el desarrollo del pensamiento crítico.

\begin{abstract}
This article arises under the project entitled "Implementation of a strategy of social appropriation of the CTeI (Science, Technology and Innovation) that promotes critical and creative thinking in children and young people from educational institutions in Tolima." Its purpose was to analyze the empirical and theoretical evidence available on the development of critical thinking, based on methodological strategies used in the classroom with elementary and secondary school students. The foregoing, after considering that critical thinking increases the participation of the educational community for the recognition and solution of problems related to the territory that is inhabited. Therefore, it is the task of the school to promote it from the earliest years; however, there are studies that show progress in the development of critical and creative thinking, but they are concentrated in higher education, while there are lags in elementary and secondary education. Finally, the reviewed research positions the role of the school as a key setting for the development of critical thinking.
\end{abstract}

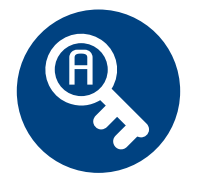

Palabras claves: Pensamiento crítico, educación, básica primaria, secundaria.

Key words: Critical thinking, education, elementary school, secondary school. 


\section{Introducción}

Este artículo se deriva del proyecto titulado "Implementación de una estrategia de apropiación social de la CteI (Ciencia, Tecnología e Innovación) que promueve el pensamiento crítico y creativo en niños, niñas y jóvenes de las instituciones educativas del Tolima”. En este contexto, se concibe la educación, la ciencia y la sociedad como tres aspectos claves que se vinculan con la apropiación social del conocimiento. Por un lado, la educación es un puente para el progreso de competencias ciudadanas porque se forman sujetos que participan activamente en la sociedad; así mismo, la CTeI, como procesos para el aprendizaje, despiertan el interés por la búsqueda de validez frente a los conocimientos; pero es importante complementarlos con saberes populares que emergen en la cotidianidad de las comunidades. Por lo anterior, se ha planteado como reto desde el proyecto macro, lograr la democratización del conocimiento a través de alternativas diversas, en las cuales el pensamiento crítico es un eje fundamental.

El desarrollo del pensamiento crítico ha sido ratificado como un mecanismo que favorece el intercambio y el diálogo reflexivo en los procesos educativos; no obstante, el modelo tradicional que domina las dinámicas en la enseñanza escolar estimula el desinterés por intercambiar conocimiento científico y de los territorios.

Por ello, el problema central planteado en el proyecto del cual se deriva este artículo es la existencia de bajos niveles de apropiación social de la ciencia, la tecnología y la innovación en las comunidades educativas (Gobernación del Tolima, Universidad de Ibagué \& Universidad del Tolima, 2018).

Dicho lo anterior, las autoras buscaron profundizar sobre el desarrollo del pensamiento crítico, teniendo en cuenta que este aumenta la participación de la comunidad educativa para el reconocimiento y solución de problemas relacionados con el territorio, por eso, es tarea de la escuela promoverlo desde los primeros años; aunque la mayoría de los estudios reportan avances en el nivel educativo superior, especialmente, y son escasos los resultados sobre los niveles de básica primaria y secundaria. En este orden de ideas, se planteó como propósito analizar la evidencia empírica y teórica disponible sobre el desarrollo del pensamiento crítico, a partir de estrategias metodológicas implementadas en el aula con estudiantes de básica primaria y secundaria.

\section{Materiales y métodos}

Para este estudio se desarrolló una revisión sucinta de la literatura que demuestra estudios asociados con incrementar los niveles de apropiación social de ciencia, tecnología e innovación en las comunidades educativas, hecho que permitió analizar publicaciones científicas del período comprendido entre el año 2005 a 2020 en las bases 
de datos Red de Revistas Científicas de América Latina y el Caribe, España y Portugal (Redalyc) y Scientific Electronic Library Online (Scielo). Los criterios para la elección de las bases de datos mencionadas se fundamentan en la divulgación de estudios provenientes, en su mayoría, de Latinoamérica y que fueran de acceso abierto.

Por su parte, la búsqueda de artículos científicos se cumplió con base en las siguientes etapas: 1) diseño de estrategia para la búsqueda bibliográfica; 2) selección de artículos de acuerdo con criterios de inclusión, entre los cuales se encuentran: documentos publicados entre los años 2012 a 2020, documentos que se refieran a preescolar, básica primaria y bachillerato. De otro lado, se incluyen documentos de revisión, análisis teóricos, de reflexión, empíricos y teóricos, artículos científicos y tesis ubicadas en repositorios académicos y, finalmente, documentos publicados en inglés o español. También, dentro de los parámetros de exclusión se encuentran: documentos publicados en años inferiores a 2012, documentos que se refieren al ciclo técnico o universitario y libros; 3 ) identificación y descripción de datos relevantes y objetivos de los artículos incluidos en la revisión; 4) análisis de contenido temático orientado a la implementación de estrategias metodológicas para el desarrollo del pensamiento crítico con estudiantes de básica primaria y secundaria.

El término central utilizado para la búsqueda en las bases de datos fue pensamiento crítico; sin embargo, con el ánimo de lograr coherencia con el propósito del proyecto macro, también se utilizaron: competencia ciudadana y competencia científica, debido a que todos están articulados. Por último, se utilizó la expresión: educación básica primaria y secundaria por los intereses planteados en esta revisión. En Scielo se encontraron dos coincidencias y en Redalyc se hallaron 23. Tras considerar los resultados de ambas búsquedas, se descartaron aquellos artículos publicados fuera del rango de 15 años de antigüedad, artículos publicados en países fuera de Latinoamérica, artículos duplicados, estudios llevados a cabo en niveles educativos diferentes a básica primaria y secundaria, artículos con conflicto de interés y que impidieran la lectura del texto completo.

Posteriormente, se cumplió con una evaluación de elegibilidad y contenido de cada texto, se descartaron nueve de ellos por presentar información incompleta, parcial o irrelevante para el objetivo de este trabajo. Las publicaciones científicas seleccionadas fueron exclusivas de la base de datos Redalyc, puesto que las dos encontradas en Scielo no cumplieron los requisitos. Finalmente, y con el propósito de analizar el contenido de cada artículo en función del objetivo planteado, se crearon categorías temáticas como resultado del proceso de su análisis previo, y en la medida en que este tipo de estrategias eran predominantes en el contenido al momento de estimular o fortalecer el desarrollo del pensamiento crítico, en consideración: 1) la argumentación, la resolución de problemas y la metacognición para el desarrollo del pensamiento crítico; 
2) la indagación, la pregunta y la resolución de problemas; 3) la didáctica mediada por las Tecnologías de la Información y la Comunicación (TIC) para el desarrollo del pensamiento crítico en el aula; 4) las competencias científicas y ciudadanas en la escuela como una ruta para el desarrollo del pensamiento crítico. En la Figura 1, se presenta el proceso de selección de los artículos científicos revisados.

Figura 1. Diagrama de flujo del proceso de selección de artículos
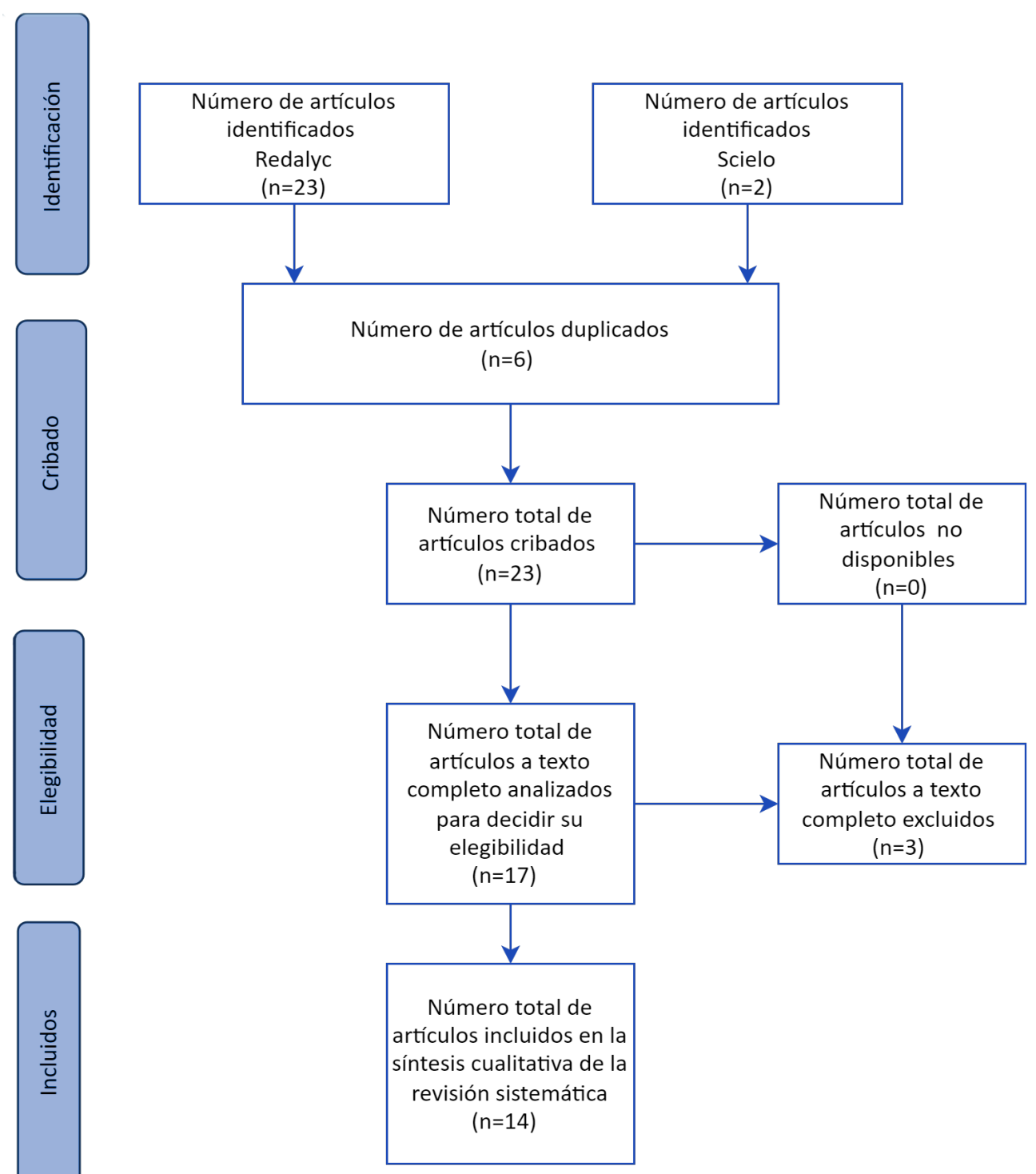

Fuente: Elaboración propia 


\section{Resultados}

El proceso de cribado implicó la selección de 14 publicaciones científicas. En la Tabla 1 se ofrece información relacionada con autores, año de publicación, país donde se ejecutó el estudio, base de datos donde fueron publicados los artículos y objetivo principal de cada uno.

Tabla 1. Resumen de artículos incluidos en la revisión

\begin{tabular}{|c|c|c|c|}
\hline Autores & Año & País & Objetivo \\
\hline $\begin{array}{l}\text { Febres Cordero, Pérez } \\
\text { \& Africano Gelves }\end{array}$ & 2017 & Venezuela & $\begin{array}{l}\text { Evidenciar la capacidad de los estudiantes para } \\
\text { reflexionar y emitir juicios. }\end{array}$ \\
\hline Rivas \& Saiz & 2016 & España & $\begin{array}{l}\text { Optimizar las habilidades intelectuales implicadas en } \\
\text { el pensamiento crítico (razonamiento, solución de } \\
\text { problemas y toma de decisiones), mediante el uso de } \\
\text { situaciones cotidianas. }\end{array}$ \\
\hline Mejía \& Mejía & 2015 & Colombia & $\begin{array}{l}\text { Comprender las relaciones entre pensamiento histórico } \\
\text { y pensamiento crítico en el marco de la enseñanza } \\
\text { de las Ciencias Sociales para la Educación Básica } \\
\text { Secundaria. }\end{array}$ \\
\hline $\begin{array}{l}\text { Calle-Álvarez \& } \\
\text { Lozano-Prada }\end{array}$ & 2018 & Colombia & $\begin{array}{l}\text { Describir las posibilidades que ofrece implementar } \\
\text { una propuesta didáctica de alfabetización digital en } \\
\text { la formación de competencias ciudadanas que den } \\
\text { sentido a la pluralidad, identidad y valoración de las } \\
\text { diferencias para estudiantes de quinto grado. }\end{array}$ \\
\hline Tito-Cordova & 2018 & Perú & $\begin{array}{l}\text { Desarrollar y fortalecer las competencias ciudadanas } \\
\text { desde las clases de Educación Física con docentes de la } \\
\text { Facultad de Educación. }\end{array}$ \\
\hline $\begin{array}{l}\text { Hernández, Arévalo \& } \\
\text { Gamboa }\end{array}$ & 2016 & Colombia & $\begin{array}{l}\text { Identificar y relacionar los niveles de competencias } \\
\text { TIC que reportan los docentes de Básica, considerando } \\
\text { su perfil de formación y tomando como referencia } \\
\text { el modelo propuesto por el Ministerio de Educación } \\
\text { Nacional (MEN) de Colombia en el año } 2013 \text {. }\end{array}$ \\
\hline Maldonado & 2018 & Colombia & $\begin{array}{l}\text { Identificar estrategias didácticas mediadas por las TIC y } \\
\text { los estilos de enseñanza para desarrollar competencias } \\
\text { ciudadanas tecnológicas. }\end{array}$ \\
\hline Tamayo Alzate & 2013 & Colombia & $\begin{array}{l}\text { Desarrollar el pensamiento crítico en estudiantes } \\
\text { de } 4 .^{\circ} \text { y } 5 .^{\circ} \text { de Básica Primaria mediante procesos } \\
\text { argumentativos efectuados por ellos en el marco de un } \\
\text { ejercicio de intervención didáctica. }\end{array}$ \\
\hline
\end{tabular}




\begin{tabular}{|c|c|c|c|}
\hline Montoya & 2007 & Colombia & $\begin{array}{l}\text { Analizar la dificultad que presentan los estudiantes } \\
\text { para pensar críticamente, las diversas concepciones } \\
\text { de pensamiento crítico y sus implicaciones para } \\
\text { responder a los retos del mundo contemporáneo. }\end{array}$ \\
\hline Guzmán Larrea & 2013 & Perú & $\begin{array}{l}\text { Mejorar la práctica pedagógica mediante la aplicación } \\
\text { de estrategias didácticas que fomenten habilidades del } \\
\text { pensamiento crítico en las clases de Ciencia, tecnología } \\
\text { y ambiente. }\end{array}$ \\
\hline $\begin{array}{l}\text { Tamayo Alzate, Zona } \\
\text { \& Loaiza }\end{array}$ & 2015 & Colombia & $\begin{array}{l}\text { Analizar las diferentes perspectivas sobre pensamiento } \\
\text { crítico y las principales categorías constituyentes del } \\
\text { mismo. }\end{array}$ \\
\hline $\begin{array}{l}\text { Moreno-Pinado \& } \\
\text { Velázquez Tejada }\end{array}$ & 2017 & España & $\begin{array}{l}\text { Contribuir al desarrollo del pensamiento crítico en los } \\
\text { estudiantes de quinto año de secundaria. }\end{array}$ \\
\hline Madrid-Hincapié & 2018 & Colombia & $\begin{array}{l}\text { Analizar las prácticas pedagógicas del grupo } \\
\text { de docentes que enseñan ciencias sociales en la } \\
\text { institución educativa Concejo de Medellín a la que } \\
\text { concurren estudiantes provenientes de sectores de } \\
\text { alta vulnerabilidad social, con las que posibilitan el } \\
\text { desarrollo del pensamiento crítico y las competencias } \\
\text { ciudadanas en los alumnos a su cargo. }\end{array}$ \\
\hline Muñoz \& Charro & 2017 & España & $\begin{array}{l}\text { Dar a conocer y ejemplificar las competencias } \\
\text { científicas que se evalúan en las pruebas internacionales } \\
\text { Programa de Evaluación Internacional de Estudiantes } \\
\text { (PISA). }\end{array}$ \\
\hline
\end{tabular}

Fuente: Elaboración propia

\subsection{La argumentación, la resolución de problemas y la metacognición para el desarrollo del pensamiento crítico}

Un análisis detallado del contenido de los estudios revisados (Febres Cordero, Pérez \& Africano Gelves, 2017; Montoya, 2007; Moreno-Pinado \& Velásquez Tejada, 2017; Rivas \& Saiz, 2016; Tamayo Alzate, 2012) apuntan a que el desarrollo del pensamiento crítico es posible, cuando se fortalece la argumentación, el razonamiento y la crítica; a la vez, se considera la resolución de problemas, en tanto el estudiante es capaz de enfrentarse a situaciones de este tipo que se vinculan a su cotidianidad; también, se activan procesos metacognitivos en la medida en que regula su propio proceso de aprendizaje.

Aun cuando existen diversidad de estrategias para fortalecer el pensamiento crítico, los estudios mencionados atribuyen una excesiva responsabilidad al estudiante, quien es el que debe responsabilizarse por el progreso de estas habilidades, opacando las discusiones sobre las prácticas pedagógicas, a través de las cuales se podría favorecer 
el aprendizaje autónomo. Lo anterior, conlleva superar el imaginario de los docentes, quienes asignan dificultades al desempeño individual de los estudiantes y generan una discusión que se centra en las prácticas pedagógicas; situación que ayudaría a superar una mirada individual de su desempeño.

Sin embargo, la responsabilidad no solo debería recaer en los docentes y los estudiantes; el sistema educativo también puede contribuir al desarrollo del pensamiento crítico, y asumir retos que impliquen la incorporación de herramientas que lleven a los diferentes actores a preguntarse sobre su rol, tanto en la escuela como en la sociedad, a través de estrategias diversas: 1) cuestionamiento continuo sobre las ideas que los estudiantes se plantean; 2) fortalecimiento en lectura comprensiva; 3) refuerzo en el proceso de razonamiento a través del lenguaje y el discurso lógico; 4) incentivo de la motivación respecto de la búsqueda del conocimiento veraz para potenciar la indagación basados en sus intereses particulares y en temas académicos o de su contexto (Febres Cordero, Pérez \& Africano Gelves, 2017; Montoya, 2007; Moreno-Pinado \& Velásquez Tejada, 2017; Rivas \& Saiz, 2016; Tamayo Alzate, 2012).

De acuerdo con Montoya (2007), es pertinente estimular en los estudiantes la posibilidad de asumir una posición autónoma que apoye las ideologías o los puntos de vista de los demás individuos; es decir, buscar caminos distintos a los ofrecidos y asumir otras perspectivas para ofrecer soluciones a los posibles problemas que tengan lugar en ese momento. Lo anterior supone espacios de participación para que exista intercambio y debate de forma respetuosa. Además, para este autor, el contexto social en el que se encuentra inmerso el estudiante es clave, en tanto se reconoce su cotidianidad y se brinda la posibilidad para transferir a este espacio los saberes académicos o científicos que se trabajan en el aula; en otras palabras, otorgar una función social al conocimiento.

Por su parte, Tamayo Alzate, Zona y Loaiza (2015) destacan la importancia de la metacognición en el desarrollo del pensamiento crítico; entendiéndose como el conocimiento que llega a tener una persona sobre sus procesos cognitivos. También se dice que es fundamental porque permite a la persona planificar, evaluar y monitorear su propio aprendizaje. Del mismo modo, los autores establecen que ella, junto con la argumentación y la solución de problemas, son aspectos centrales y por tener en cuenta para el logro de un adecuado avance en el desarrollo del pensamiento crítico a lo largo del proceso de formación en la escuela.

En este mismo camino, la resolución de problemas se utiliza como estrategia didáctica para que el estudiante encuentre alternativas a situaciones específicas; de modo que su relación con el pensamiento crítico se daría en el momento en que el primero identifica el grado de complejidad del mismo y sus posibles alternativas de solución, mientras que el segundo tendría una función selectiva de alternativas apropiadas para resolver situaciones de su entorno (Tamayo Alzate, Zona \& Loaiza, 2015). 
Por su parte, Mejía y Mejía (2015) llevaron a cabo un estudio cuyo propósito fue comprender la relación que existe entre el pensamiento crítico y el pensamiento histórico en el marco de la enseñanza en el área de Ciencias Sociales, encaminada al ciclo de educación en básica secundaria. Para ello consideraron la solución de problemas, la argumentación y la metacognición como estrategias de aprendizaje que favorecen el pensamiento histórico a través del desarrollo de habilidades como la empatía, la relación presente-futuro, presente, relación pasado remoto-presente, permanencia o continuidad y, finalmente, cambio. En este sentido, sugieren incluir el pensamiento crítico como nueva categoría para entender el pensamiento histórico, debido a su estrecha relación $\mathrm{y}$ a su potencial para abordar las lecturas comprensivas desde el pasado, el presente y el futuro.

Así mismo, Tamayo Alzate (2012) presentó un estudio en Colombia con 2200 estudiantes de cuarto y quinto grado de educación básica primaria, pertenecientes a instituciones educativas públicas. Su objetivo fue posibilitar el desarrollo del pensamiento crítico de los participantes mediante una intervención con diez actividades conducentes hacia la argumentación. Los resultados ratificaron desde las estructuras argumentativas empleadas por los niños en cada actividad, que existe una estrecha relación entre la metacognición y la argumentación; esto quiere decir que cuando el sujeto reconoce sus propios procesos cognitivos, aprende a regularlos, evaluarlos y monitorearlos. En otras palabras, la activación de procesos metacognitivos posibilita a cada individuo regular, evaluar, monitorear y reconocer sus debilidades y fortalezas frente a sus competencias argumentativas (Tamayo Alzate, 2012).

Lo expuesto ratifica que la misión de la escuela no debe basarse en transmitir al alumno una infinidad de conocimientos terminados, absolutos e indiscutibles, sino, ante todo aprender a aprender, procurar que el alumno llegue a adquirir una autonomía intelectual y pueda utilizar estos conocimientos para resolver problemas asociados a su cotidianidad; esto es, ratificar que los conocimientos tengan una función social. Así las cosas, es urgente focalizar esfuerzos hacia el avance de destrezas de orden superior como las del pensamiento crítico, pues su progreso va más allá del entrenamiento de habilidades cognitivas e incluso distingue disposiciones de la persona, que aporta a una tarea de pensamiento en función de rasgos como la apertura mental, la sensibilidad hacia las creencias, los sentires y saberes ajenos; así como las formas posibles para enfrentar retos de la vida.

\subsection{La indagación, la pregunta y la resolución de problemas}

Para el desarrollo del pensamiento crítico, Montoya (2007) sugiere a los maestros incitar o tensar las ideas y saberes a través de la pregunta. El cuestionamiento exige razonar e intercambiar ideas gracias al lenguaje; también posibilita navegar en el conocimiento mediante la indagación y la problematización de la realidad. 
Por lo anterior, el docente mediante su práctica, dispone de escenarios donde los conceptos, la cotidianidad, entre otros, son analizados desde múltiples lentes; de allí emergen alternativas de solución como producto del ejercicio de reflexión en el cual se involucra el estudiante. Dialogar bajo estas condiciones implica reconocer al otro sin absolutismos; respetar las ideas pese a que no coincidan con algunos o con la mayoría.

En suma, es un ejercicio que armoniza el espacio y las relaciones entre los educandos y el educador porque se privilegia la organización de entornos cooperativos y solidarios en los cuales tienen lugar el contexto social y la identidad cultural para viabilizar juicios e ideas.

Por su parte, Febres Cordero, Pérez y Africano Gelves (2017) proponen las comunidades de aprendizaje como estrategia alternativa para que el pensamiento en el aula aflore; se trata de un espacio que se opone al tradicionalismo educativo porque educandos y educadores exteriorizan sus ideas, las comparten y las reconocen debido a la riqueza que aportan para la construcción o reconstrucción de saberes.

En las comunidades de aprendizaje se acude a las destrezas, habilidades, experiencias y competencias de los actores que participan. Así las cosas, las ideas se deben comunicar de forma clara y efectiva, hacer uso de analogías, pensar sin olvidar las implicaciones y consecuencias prácticas que emergen, emitir juicios argumentados y formular preguntas claras que puedan llegar a ser discutidas. Un ejemplo es la comunidad de aprendizaje denominada "Gestión y Socialización del conocimiento de Fundacite Mérida”, allí, sus integrantes piensan de forma crítica con miras a fortalecer su desarrollo intelectual y académico (Febres Cordero, Pérez \& Africano Gelves, 2017).

En concordancia, en Lima (Perú), Moreno-Pinado y Velásquez Tejada (2017) retoman un estudio previamente realizado con 42 estudiantes de quinto de secundaria, cuyo objetivo fue identificar cómo analizan los estudiantes la información cuando se enfrentan a una situación nueva. Los resultados evidencian que el ejercicio de análisis es precario; por ejemplo, los niños no plantean alternativas de solución y, más bien, tienden a reproducir ideas. En este mismo contexto, la forma como los profesores aproximan a los estudiantes a un saber dificulta el desarrollo del pensamiento crítico porque tienden a esperar respuestas enciclopédicas o textuales. Esta situación motivó al autor del estudio a diseñar una estrategia didáctica fundamentada en perspectivas socioeducativas, pedagógicas y curriculares, en las que se vinculan procesos de enseñanza-aprendizaje que contemplan la dimensión afectiva, motivacional y metacognitiva, para así potenciar las habilidades y capacidades del pensamiento que poseen los estudiantes en todas las formas posibles.

Para lo anterior, se desarrollaron variadas actividades organizadas por fases, estas fueron: sensibilización, organización, ejecución y evaluación. Cada una se estructuró de tal forma que los estudiantes se sintieran retados a razonar de forma 
lógica; reflexionar sobre el conocimiento que poseen frente al que están adquiriendo y asumir desde el trabajo cooperativo la construcción de sentido de pertenencia (Moreno-Pinado \& Velásquez Tejada, 2017). En esta misma línea, Guzmán Larrea (2013) diseñó un programa de intervención, con el fin de fortalecer las prácticas pedagógicas de los maestros. Para lo anterior, se utilizaron estrategias didácticas que promovieran el desarrollo de habilidades asociadas al pensamiento crítico en los cursos de ciencia, tecnología y medio ambiente.

Dentro de las propuestas, se encuentran las de tipo experiencial, esto quiere decir que exigen trasladar lo visto en clase a la práctica; por ejemplo, los laboratorios, las sesiones que incluyen espacios para preguntas, debates o propuestas por parte de los estudiantes, entre otros. Así las cosas, el docente tiene un papel de escucha atenta para estimular el diálogo a través de preguntas motivadoras en pro del análisis de dificultades articuladas con el contexto. Por ejemplo, habilidades matemáticas, lectura cuidadosa y comprensiva, análisis de un problema y propuesta de soluciones innovadoras. Todo lo anterior se aborda especialmente en los equipos de trabajo cooperativo, en los cuales los estudiantes se enfrentan a retos que les exige explorar su capacidad creativa y el despliegue de habilidades científicas (Guzmán Larrea, 2013).

A partir de lo expuesto, surgen tres cuestiones: 1) ¿cómo en los procesos de formación inicial de los futuros docentes se aporta al desarrollo de su pensamiento crítico? de manera que puedan promoverlo en el aula a través de las prácticas de enseñanza; 2) ¿cómo se asume el conocimiento en la escuela?; 3) ¿los docentes y los estudiantes se dan la oportunidad de dudar sobre supuestas verdades? $\mathrm{o} i$ consumen ideas como si fueran absolutas? Ante este panorama, el problema no es la enseñanza de conocimientos en la escuela, sino las limitaciones para reconstruir saberes sobre esos conocimientos abordados, de forma que los estudiantes puedan hacer uso de ellos para trasladarlos a su cotidianidad. Se reitera la cuestión sobre cómo lograr que, en la escuela, el conocimiento tenga una función social. En este sentido, es fundamental ayudar a los estudiantes a desarrollar competencias mediante el conocimiento y el reconocimiento de diversas ideas, indagar sobre ellas, reflexionar y no conformarse con aprender a manejar la información que llega a sus manos.

\subsection{La didáctica mediada por las TIC para el desarrollo del pensamiento crítico en el aula}

Hoy en día el uso de las TIC se ha convertido en un medio fundamental para el diseño de herramientas que faciliten la gestión en el aula con miras a enseñar y promover la creatividad y la innovación. Estas tecnologías ayudan a que el entorno educativo esté cambiando, lo cual favorece la enseñanza y la apropiación de conocimientos diversos. Por lo anterior, su uso educativo fomenta el despliegue de actitudes que conducen 
al aprendizaje de la ciencia y la tecnología, mediante el intercambio de ideas en un escenario donde aflora la motivación y el interés por la ciencia (Maldonado, 2018).

La mediación de las TIC para el desarrollo del pensamiento crítico se distingue como oportunidad para estar a la vanguardia con nuevos entornos en los cuales los estudiantes muestran necesidades diferentes que van de la mano con los cambios propios del siglo XXI. Así las cosas, su uso y la implementación de la cultura digital en el proceso educativo son una condición necesaria para transformar las prácticas pedagógicas con estrategias aplicables en el aula para el pensamiento crítico, debido a que crean la posibilidad de aplicación posterior en el contexto (Maldonado, 2018).

Algunas investigaciones sobre destrezas docentes que implican el desarrollo de la competencia técnica o digital señalan que el uso de las nuevas tecnologías son la base para la mejora de nuevas destrezas, pues ellas le permiten al educador reflexionar alrededor de cuestiones como ¿saber qué? ¿saber cómo? y ¿cuándo hacerlo? Por lo tanto, las competencias digitales se entienden como un conjunto de habilidades y conocimientos básicos para utilizar las TIC y para afrontar nuevos retos sociales en el ámbito académico, de formación y conocimiento digital (Calle-Álvarez \& LozanoPrada, 2018).

El desarrollo de tales competencias digitales facilita a los educadores su papel en la implementación de las TIC con un objetivo pedagógico claro; Hernández, Arévalo y Gamboa (2016) refieren que muchos docentes son considerados como inmigrantes digitales, esto significa que a menudo utilizan la tecnología con fines instrumentales; en otras palabras, en búsqueda de diferentes tipos de información, actividades de ocio y entretenimiento, pero necesitan mejorar su capacidad de procesamiento para estar en el aula.

Es evidente que el docente es un agente central para el correcto uso de las TIC en el proceso educativo, puesto que, es él quien decide incorporarlas y de qué manera, así, "... al prepararse para brindar servicios a los estudiantes, los profesores también son responsables de diseñar oportunidades de aprendizaje y entornos propicios en el aula para promover que los estudiantes utilicen las TIC para aprender y comunicarse" (UNESCO, 2008, p. 2).

Lo anterior sugiere como necesidad establecer diagnósticos para comprender la realidad actual de los docentes en educación básica respecto de sus competencias TIC y sobre las políticas de formación inicial y permanente en los diferentes centros educativos. Todo esto supone proponer nuevas pautas de formación permanente e incentivarlos a mejorar, actualizar y consolidar sus conocimientos a fin de aplicar tecnología (Hernández, Arévalo \& Gamboa, 2016).

En suma, las TIC se han unido a la educación y han dado como resultado métodos de enseñanza innovadores que se adaptan a los nuevos modelos de formación 
y a las nuevas realidades de los educandos, así, constituyen una alternativa mediadora para dejar atrás rasgos tradicionales basados en la difusión de conocimientos absolutistas, y dar paso a la organización de ambientes de aprendizaje colaborativos y participativos.

\subsection{Las competencias científicas y ciudadanas en la escuela: una ruta para el desarrollo del pensamiento crítico}

Como se mencionó en el apartado anterior, un docente motivado por su proceso de formación busca actualizarse y enseñar desde los saberes pedagógicos y cotidianos. Dentro de las exigencias normativas para el desarrollo del pensamiento crítico se resaltan, desde los primeros años escolares, las competencias científicas que aluden a ¿cómo orientar procesos educativos hacia este tipo de competencias? Al respecto, han señalado Muñoz y Charro (2017) en los ítems del PISA tres competencias científicas, estas son: 1) evaluar y diseñar la investigación científica; 2) interpretar datos y pruebas científicas; 3) explicar los fenómenos científicamente.

La primera corresponde con la evaluación y diseño de la investigación científica; es decir, se hace referencia al conocimiento procesal en investigación y los estudiantes se involucran con actividades que exigen el despliegue de habilidades tales como identificar preguntas que pueden ser estudiadas, distinguir entre preguntas que la ciencia puede y no puede responder, identificar variables y categorías que se pretenden investigar, entre otras.

La segunda equivale a la interpretación de datos y el uso de pruebas científicas. En este caso, PISA plantea a los estudiantes el análisis y la interpretación de datos científicos; lo anterior alude a tomar evidencias, afirmaciones y argumentos presentados mediante diversas formas de abstracción (descripciones de texto, tablas, diagramas de ejes de coordenadas) en lugar de coordenadas, imágenes o gráficos. Lo anterior facilita identificar conclusiones sobre el fenómeno estudiado.

La tercera está vinculada con la explicación científica de los fenómenos revisados, que exige a los estudiantes procesar el contenido, el procedimiento y el análisis de los resultados a la luz de un marco teórico; sin embargo, Muñoz y Charro (2017) ponen en cuestión ¿cómo pueden lidiar los estudiantes con los datos recolectados?, pues ellos enfocan su atención en la evaluación porque pueden analizar e interpretar datos científicos, evidencias, afirmaciones y argumentos presentados en una variedad de formas abstractas (por ejemplo, descripciones de texto, tablas, diagramas de ejes, etc.), coordenadas, imágenes o gráficos para obtener conclusiones adecuadas sobre el fenómeno o situación. Por consiguiente, esta competencia sigue requiriendo, en mayor medida, el uso del conocimiento procedimental centrado en el análisis de los datos. 
Adicionalmente, Muñoz y Charro (2017) cuestionan ¿cómo los estudiantes comprenden o analizan conceptos científicos contenidos en el fenómeno estudiado?, debido a que podrían estar en la capacidad de usar el conocimiento y las habilidades científicas para comprender el mundo natural y, particularmente, para intervenir y tomar decisiones que afecten estos entornos (Muñoz \& Charro, 2017). Se reitera que, a través de la enseñanza, son ellos quienes deben fortalecer las habilidades necesarias para resolver situaciones de su contexto, gracias a los conocimientos revisados y reconstruidos para garantizar su función social. Luego, las políticas educativas dirigidas a la calidad deben requerir la formación calificada gracias a su participación en programas de formación por competencias; así las cosas, el educador que plantea a sus educandos problemas de investigación, preguntas y situaciones retadoras y motivantes, sitúa al sujeto como un pensador crítico.

Por otro lado, las competencias en educación media que se enmarcan en el método de la reflexión crítica (Tito Cordova, 2018) se sitúan hacia la formación de ciudadanos que interactúan con símbolos que son producto de construcciones humanas, a través de las cuales el lenguaje opera como mediador para intercambiar ideas y decidir qué hacer y qué no, cómo tratar el contenido, la fuente y el método; en otros términos, el desarrollo del pensamiento crítico proporciona criterios suficientes para que las personas valoren diversas fuentes de información y asuman una postura argumentada para comprender y resolver un problema escolar y del día a día.

De acuerdo con Tito Cordova (2018), los educadores que aportan al desarrollo de competencias ciudadanas desde la formación del pensamiento crítico de sus estudiantes, organizan experiencias educativas en las que todos colaboran, racionalizan y reflexionan para decidir qué hacer o creer (Calle-Álvarez \& Lozano-Prada, 2018), esta interacción implica hacer uso de habilidades metacognitivas (Tamayo Alzate, Zona \& Loaiza, 2015) porque ayudan al desarrollo de la capacidad de analizar, reflexionar, valorar los propios pensamientos y expresarlos (Moreno-Pinado \& Velásquez Tejada, 2017).

Ahora bien, las competencias ciudadanas emergen gracias a los conocimientos situados y cuestionables, a las cogniciones, emociones y habilidades comunicativas, que combinados entre sí posibilitan acciones constructivas en una sociedad democrática (Madrid-Hincapié, 2018). En palabras de Madrid-Hincapié (2018), el pensamiento crítico fomenta el respeto y el cuidado de los otros; por un lado, la voz de todos y no de algunos es importante $y$, por otro, todos los individuos participantes son tratados como seres humanos con derechos y voz propia. Al respecto, Parra (como se cita en MadridHincapié, 2018) alude lo siguiente: 
La cultura escolar es tal vez el instrumento pedagógico más complejo y poderoso con que cuenta la escuela, no solo para llevar a cabo su trabajo en el campo del conocimiento, sino fundamentalmente para ejercer su labor de formadora de ciudadanos. La cultura escolar se expresa en la vida cotidiana y es en esa vida, y no en los discursos escolares sobre la democracia, donde se crean y refuerzan valores, normas y formas de ver y vivir la vida. La eficacia pedagógica de la cultura es notable: si la escuela tiene una organización social, una cultura autoritaria, estará formando ciudadanos para el mantenimiento de la sociedad autoritaria. Si la escuela vive una cultura democrática real estará entonces formando ciudadanos para la vida democrática. (p. 8).

\subsection{Recapitulaciones}

Los estudiantes se deben centrar en la capacidad de usar sus conocimientos para afrontar los retos de la vida real; no se trata solo de adquirir nuevos saberes, sino de interiorizarlos y reconstruirlos. Las competencias ciudadanas estimulan su interés por el aprendizaje; a la vez potencian el desarrollo de habilidades. Esto se puede lograr por medio de la participación activa en proyectos aplicables a sus comunidades, de manera que puedan comprometerse con el proceso educativo y así otorgar una función social al conocimiento. Además, cuando los docentes estimulan los procesos de aprendizaje de los estudiantes y los animan a desarrollar habilidades cívicas, les posibilitan ser agentes de cambio social.

El desarrollo del pensamiento crítico en las aulas latinoamericanas durante los últimos años presenta limitantes y solo en casos aislados logra evidenciarse el interés de los maestros o instituciones educativas que buscan despojarse del tradicional proceso de enseñanza-aprendizaje, para responder a las demandas del mundo actual; esto implica pensar sobre cómo se están formando los futuros docentes y cómo se transforman, revisan y evalúan sus prácticas pedagógicas.

Los estudios revisados ponen en evidencia que el pensamiento crítico se ha implementado gradualmente en la formación docente y los profesores logran direccionar sus procesos de enseñanza hacia el incremento de estas habilidades en ellos. El uso y aplicación de la TIC no debe ser un tema aislado a la generación de pensamiento crítico; por el contrario, en el mundo actual sirve como medio o herramienta para promover el mismo, en tanto se fortalecen la criticidad, el manejo de la información; su validez, veracidad y se toman decisiones.

La resolución de problemas como estrategia para potenciar el pensamiento crítico aporta al estudiante en el sentido que promueve en él, la constante búsqueda de diferentes alternativas. Así se logra que no solo las dificultades académicas sean resueltas a partir de diferentes posibilidades, sino que también usen la estrategia con los inconvenientes de su vida cotidiana. Por su parte, las preguntas que surgen en el estudiante y la posibilidad de ser resueltas apropiadamente por el docente conllevan 
en el primero, la capacidad de seguir indagando sobre todo aquello que no logra comprender por completo y resolver sus propios interrogantes.

Como estrategia, las preguntas desafían a los estudiantes a confrontarse, a analizar situaciones, a desarrollar su capacidad de búsqueda, a generar alternativas, a considerar el contexto, a buscar más allá de lo que parece una verdad absoluta, a comprender a profundidad un fenómeno. También contribuye a fortalecer habilidades para identificar, clasificar y plantearse inquietudes para hallar posibles soluciones. Las instituciones educativas podrían brindar a sus docentes la formación necesaria para que puedan implementar en su plan pedagógico o en sus estrategias de enseñanza, la promoción del pensamiento crítico en los estudiantes, en relación con sus necesidades y contexto.

Es importante que los docentes reconozcan con mayor fuerza las ventajas del desarrollo del pensamiento crítico en sus estudiantes, sus potencialidades y las diferentes variantes que maneja. Los estudios coinciden en que el pensamiento crítico fortalece otras áreas y habilidades como el pensamiento científico, el pensamiento ciudadano, la argumentación, la resolución de problemas, la metacognición, la indagación, la apropiación y la utilización del mismo junto con las TIC, facilitadoras en el desempeño académico y social.

Así mismo, las investigaciones develan algunas fallas en la implementación de estrategias para el desarrollo del pensamiento crítico, y aunque en muchos países europeos y del continente americano se ha reconocido su importancia e impacto en los procesos de aprendizaje, aún son escasas las evidencias de sus aplicaciones. La responsabilidad recae en la labor docente y olvida que los procesos de enseñanza y aprendizaje se configuran a través de la flexibilidad de políticas institucionales y proyectos que aporten al pensamiento crítico.

Es necesario fomentar espacios por medio de los cuales los docentes puedan proponer y liderar estrategias, aprovechar sus potencialidades, construir equipos con otros colegas para explorar experiencias y valorar acciones de su quehacer. La escuela y todos sus actores deben involucrarse de forma específica en cada una de las acciones que contribuyen al desarrollo del pensamiento crítico. Por lo tanto, es necesario comprender las realidades de los educandos, las necesidades formativas de quienes los orientan en la academia; que involucren a los padres de familia y directivos en general. Desde luego, potenciar prácticas educativas democráticas que propendan por una formación integral y que incluyan a todos los responsables del proceso.

Los profesores deben generar ambientes participativos para promover el rol activo de los estudiantes. En este sentido organizar espacios para que asuman retos, investiguen, se cuestionen y se interesen por su propio proceso de aprendizaje, desde una mirada más experiencial y crítica. Las investigaciones contemplan la necesidad 
de explorar una dimensión sensible y emotiva; de manera que en conjunto con el profesor y el padre de familia se reconozcan como sujetos importantes y necesarios en el funcionamiento de la escuela y de todos los procesos de enseñanza y aprendizaje que se tejen allí.

Es imperante una función del docente como mediador en todos los procesos de aprendizaje y no desde la idea de imposición. En síntesis, permitir espacios democráticos en los cuales se intercambien saberes, cuestiones, dudas, se hagan aclaraciones y se le conceda al estudiante encontrar a través de las experiencias de otros y propias, soluciones aterrizadas.

Las habilidades de pensamiento crítico deben desarrollarse de manera transversal, estar presentes a lo largo del currículo y abordarse en todas las dimensiones y áreas; por tanto, es tarea de los directivos y docentes trabajar conjuntamente, fomentar estrategias para posibilitar el intercambio de conocimiento, que el estudiante entienda un problema desde las distintas dimensiones, que sea capaz de elegir, exponer sus ideologías, valorar los pensamientos de otros sin prejuicios sociales y responsabilizarse de sus actos. A la vez, asumir las decisiones que se toman en la escuela como oportunidades para potenciar su proyecto de vida y funcionar en sociedad.

Argumentar con razones válidas ayuda al estudiante a establecer un criterio, para tomar postura y decisiones ante diferentes situaciones que se presenten en la cotidianidad. Como herramienta de comunicación, favorece el pensamiento crítico y lleva a cada uno a enfrentar los desafíos de su realidad más próxima desde la comprensión y complejidad de los distintos fenómenos. En este sentido se potenciará en ellos la capacidad para razonar, involucrarse y difundir sus ideas después de una evaluación minuciosa de todos los factores.

\section{Potencial uso}

Los resultados de esta revisión develan la necesidad de aumentar el número de estudios en Colombia y en el Tolima, específicamente, a partir de los cuales se muestren avances o rutas pedagógicas para promover el pensamiento crítico en la escuela desde los primeros años, debido a que la mayor parte de las investigaciones se concentran en la educación superior, mientras hay rezagos en la educación primaria y secundaria. 
Título del proyecto que lo deriva o vincula: Implementación de una estrategia de apropiación social de la CTeI que promueva el pensamiento crítico y creativo en niñas, niños y jóvenes de las instituciones educativas del Tolima.

PRIT: Educación y bienestar para el desarrollo humano integral. Código: 19-519-ESP.

Ficha técnica del proyecto
Palabras claves: Pensamiento crítico, educación, básica primaria y secundaria.

Grupo de investigación: Grupo de Estudios en Educación, GESE. Investigadora principal: Laura Yamile Henao.

Correo electrónico: laura.henao@unibague.edu.co

\section{Referencias}

Calle Álvarez, G. Y., \& Lozano-Prada, A. (2018). La alfabetización digital en la formación de competencias ciudadanas en la básica primaria. Eleuthera, 20(1), 35-54. Recuperado de http://www.scielo.org.co/pdf/eleut/v20/2011-4532-eleut-20-00035.pdf

Febres Cordero, C., A., Pérez, Á. A., \& Africano Gelves, B. B. (2017). Las pedagogías alternativas desarrollan el pensamiento crítico. Educere, 21(69), 269-274. Recuperado de https://www.redalyc.org/journal/356/35655222005/html/

Gobernación del Tolima, Universidad de Ibagué \& Universidad del Tolima. (2018). Documento técnico "Implementación de una estrategia de apropiación social de la CTeI que promueva el pensamiento crítico y creativo en niños, niñas y jóvenes de las IE del Tolima”. Código BPIN: 2017000100049.

Guzmán Larrea, R. E. (2013). Estrategias didácticas que fomentan habilidades del pensamiento crítico. UCV-HACER. Revista de Investigación y Cultura, 2(1), 178-188. Recuperado de https://www.redalyc.org/articulo.oa?id=5217/521752180021

Hernández, C., Arévalo, M., \& Gamboa, A. (2016). Competencias TIC para el desarrollo profesional docente en educación básica. Praxis \& Saber, 7(14), 41-69. https://doi.org/10.19053/22160159.5217

Madrid-Hincapié, J. M. (2018). Prácticas pedagógicas del docente de ciencias sociales para el desarrollo del pensamiento crítico y competencias ciudadanas en contextos de vulnerabilidad social. Revista Latinoamericana de Estudios Educativos, 14(1), 150-168. Recuperado de https://www.redalyc.org/jatsRepo/1341/134156702009/html/index.html

Maldonado, M. E. (2018). El aula, espacio propicio para el fortalecimiento de competencias ciudadanas y tecnológicas. Sophia, 14(1), 39-50. Recuperado de https://www.redalyc.org/jatsRepo/4137/413755833004/index.html

Mejía, L., \& Mejía, A. (2015). Relaciones entre pensamiento histórico y pensamiento crítico en la enseñanza de las ciencias sociales en estudiantes de educación básica secundaria. Revista Interamericana de Investigación, Educación y Pedagogía, 8(2), 413-436. Recuperado de https://www.redalyc.org/pdf/5610/561058729010.pdf 
Montoya, J. I. (2007). Primer avance de investigación. Acercamiento al desarrollo del pensamiento crítico, un reto para la educación actual. Revista Virtual Universidad Católica del Norte, 1(21), 1-17. Recuperado de https:/www.redalyc.org/pdf/1942/194220390001.pdf

Moreno-Pinado, W. E., \& Velázquez Tejeda, M. E. (2017). Estrategia didáctica para desarrollar el pensamiento crítico. REICE. Revista Iberoamericana sobre Calidad, Eficacia y Cambio en Educación, 15(2), 53-73. Recuperado de https://www.redalyc.org/pdf/551/55150357003.pdf

Muñoz, J., \& Charro, E. (2017). Los ítems PISA, una herramienta para la identificación de las competencias científicas en el aula. Revista Electrónica en Educación y Pedagogía, 1(1), 106-122. https://doi.org/10.15658/rev.electron.educ.pedagog 17.09010107

Rivas, S. F., \& Saiz, C. (2016). Instrucción en pensamiento crítico: influencia de los materiales en la motivación y el rendimiento. Revista Latinoamericana de Estudios Educativos (Colombia), 12(1), 91-106. Recuperado de https://www.redalyc.org/pdf/1341/134149742006.pdf

Tamayo Alzate, O. E. (2012). La argumentación como constituyente del pensamiento crítico en niños. Hallazgos, 9(17), 211-233. Recuperado de https://www.redalyc.org/pdf/4138/413835215010.pdf

Tamayo Alzate, O. E., Zona, R., \& Loaiza, Y. E. (2015). El pensamiento crítico en la educación algunas categorías centrales de su estudio. Revista Latinoamericana de Estudios Educativos (Colombia), 11(2), 111-133. Recuperado de https://www.redalyc.org/pdf/1341/134146842006.pdf

Tito Cordova, L. (2018). Competencias ciudadanas desde las clases de Educación Física en las Facultades de Educación. Horizonte de la Ciencia, 8(15), 133-147. Recuperado de https://www.redalyc.org/jatsRepo/5709/570960688011/movil/index.html

UNESCO. (2008). Estándares de competencias TIC para docentes. Recuperado de http://www.oei.es/tic/UnESCOEstandaresDocentes.pdf 